\title{
Effect of ethanol-wet bonding on porosity and retention of fiberglass post to root dentin
}

\section{Monize Ferreira Figueiredo de CARVALHO(a) Monica YAMAUTI(b) (D) \\ Cláudia Silami de MAGALHÃES(b) \\ Aline Aredes BICALHO(c) (D) \\ Carlos José SOARES(d) (iD \\ Allyson Nogueira MOREIRA(e)}

(a) Faculdade de Sete Lagoas - Facsete, Department of Restorative Dentistry, Sete Lagoas, MG, Brazil.

(b) Universidade Federal de Minas Gerais UFMG, School of Dentistry, Department of Restorative Dentistry, Belo Horizonte, MG, Brazil.

(c) Universidade Federal de Uberlândia - UFU, Technical Health School, Uberlândia, MG, Brazil.

(d) Universidade Federal de Uberlândia UFU, School of Dentistry, Department of Operative Dentistry and Dental Materials, Uberlândia, MG, Brazil.

(e) Universidade Federal de Minas Gerais UFMG, School of Dentistry, Department of Restorative Dentistry, Belo Horizonte, Minas Gerais, Brazil.

Declaration of Interests: The authors certify that they have no commercial or associative interest that represents a conflict of interest in connection with the manuscript.

\section{Corresponding Author:}

Monize Ferreira Figueiredo de Carvalho

E-mail: monize_c@hotmail.com

hitps://doi.org/10.1590/1807-3107bor-2020.vol34.0020

Submitted: April 11, 2019

Accepted for publication: December 16, 2019

Last revision: February 3, 2020
Abstract: This in vitro study aimed to assess the effects of different pretreatments used to adapt glass-fiber posts cemented to root canals with different resin cements, regarding porosity percentage and bond strength. Twelve bovine incisor roots were prepared with Largo drills. After post space preparation, the specimens were randomized into two types of pretreatment groups $(n=3)$ : water-wet bonding and ethanol-wet bonding. After the post were cemented, the roots were stored in $100 \%$ humidity at room temperature for 7 days. The samples were scanned by microcomputed tomography (micro-CT). Images were reconstructed by NRecon software, and CTAn software was used to analyze the porosity percentage (\%) at the luting interface. Evaluation of the push-out bond strength was performed by serially cutting the roots, and submitting the slices to testing. Additionally, the resin cement post-gel shrinkage values (\%) were measured using the strain-gauge method $(n=10)$. Data were analyzed by two-way ANOVA, Tukey's test and Student's $t$ test $(a=0.05)$. The roots prepared with ethanol-wet bonding using RelyX ${ }^{\mathrm{TM}} \mathrm{U} 200$ had significantly lower porosity in the coronal and apical thirds $(p<0.05)$. The group prepared with ethanol-wet bonding using RelyX ${ }^{\mathrm{TM}}$ ARC presented better bond strength results in the coronal and apical thirds $(\mathrm{p}<0.05)$. Rely $\mathrm{X}^{\mathrm{TM}}$ ARC $(0.97 \%)$ produced a higher post-gel shrinkage value than RelyX ${ }^{\mathrm{TM}} \mathrm{U} 200$ $(0.77 \%)$. Canals pretreated with ethanol-wet bonding presented better outcomes in regard to porosity percentage and push-out bond strength.

Keywords: Dental Bonding; X-Ray Microtomography; Resin Cements.

\section{Introduction}

The rehabilitation of endodontically treated teeth may be different from that of vital teeth, despite alteration of the coronal tooth structure. Teeth with fewer than two coronal walls usually require additional specialized retention. ${ }^{1}$ Intraradicular posts are needed to provide retention if the remaining coronal tooth structure is not sufficient. ${ }^{2}$ Glass-fiber posts have properties that are suitable for restoring endodontically treated teeth. They have high flexural strength and an elasticity modulus (20 GPa) similar to that of dentin (18 GPa), ${ }^{2}$ resulting in lower stress concentration at the root, and thus reducing the risk of root fractures. ${ }^{1}$ However, these posts are susceptible to failure. The main reason is 
debonding, especially at the cement-dentin interface, considered the most vulnerable link. ${ }^{3}$ Indeed, some factors can influence the retention of posts in root canals, such as density and orientation of dentinal tubules, ${ }^{4}$ root-canal geometry, ${ }^{5}$ and difficulty in light-activating the luting material. ${ }^{3}$

The instability of resin-dentin bonds has been attributed to the degradation of hybridized dentin, resin and collagen fibrils. This degradation can occur due to hydrolytic degradation of the resin by water sorption, or to enzymatic degradation of exposed collagen fibrils. ${ }^{6}$ Hence, it is fundamental to find an alternative to glass-fiber posts, which can influence the longevity of postendodontic restorations and increase the longevity of bonding. ${ }^{7}$ Ethanol-wet bonding has been proposed by Pashley et al. ${ }^{8}$ as a possible alternative. The authors reported the implications of using ethanol to replace water in the demineralized collagen matrix, and of infiltration of relatively hydrophobic monomer resins in demineralized dentin, leading to the formation of hydrophobic hybrid layers. This bonding technique can prevent water from forming interpeptide $\mathrm{H}$-bonds. Consequently, the dentin matrix can expand with relatively wide interfibrillar spaces, providing maximal monomer uptake during the infiltration of solvated comonomers. This technique creates hydrophobic hybrid layers that absorb less water and generate durable resin-dentin bonds. ${ }^{8}$

Ethanol-wet bonding was initially developed for etch and rinse adhesive systems, ${ }^{8}$ in which resin monomers penetrate a delicate layer of unsupported collagen fibrils exposed to an etching agent. ${ }^{9}$ This bonding technique was indicated for this type of adhesive to prevent against the degradation of non-protected demineralized dentin $(5 \mu \mathrm{m})$ beneath the hybrid layer. ${ }^{10}$ In cases of self-etching adhesives, the unsupported collagen fibrils are thinner (0.5-1.5 $\mu \mathrm{m}$ deep $)^{8}$ due to the mechanism of action of ionic resin monomers, which modify and integrate the hybrid layer simultaneously. ${ }^{10}$

Resin cements are classified into two categories, according to the bonding strategy: dual cure resin cement and self-adhesive cement. Dual-cure resin cements are characterized by the use of a preliminary bonding agent, and are classified as: etch-and-rinse, self-etching or universal adhesives systems. ${ }^{11}$ Comparatively, the multi-step application technique is time-consuming and somewhat technique-sensitive. In brief, self-adhesive resin cements have simplified the indirect luting technique by eliminating any pretreatment of the dentin surface. ${ }^{11}$ The composition of this material is based on acid monomers, which are responsible for demineralizing and infiltrating the substrate, thus creating micromechanical retention and chemical bonding to hydroxyapatite. ${ }^{3}$ However, the hybrid layer penetrates the cement in radicular dentinal tubules only slightly. ${ }^{6}$ Overall, the literature on the efficacy of self-adhesive resin cements bonding to root canal dentin concerning ethanol-wet bonding is scarce. ${ }^{6}$ Obtaining a durable resin-dentin interface is crucial for the rehabilitation of endodontically treated teeth using glass-fiber posts. The simplified ethanol-wet bonding technique revealed promising results with respect to bond strength stability of the glass-fiber post inside the root canal. ${ }^{7}$

The aim of this in vitro study was to evaluate the adaptation of glass-fiber posts cemented to root canals by two different pretreatments, regarding the percentages of total porosity at interfaces (\%), bond strength (MPa) and post-gel shrinkage values (\%) of the tested resin cements. The null hypotheses were as follows: a) that the total porosity percentages would not affect the different pretreatments in different portions of the root; $b$ ) that the bond strength would not be affected by the different protocols in different portions of the root; and c) that the resin cements would have similar post-gel shrinkage values.

\section{Methodology}

\section{Sample selection and root canal preparation}

A convenience sample was performed based on studies using micro-CT methodology. ${ }^{22,24} \mathrm{~A}$ total of 12 freshly extracted bovine incisors of homogeneous shape and size were obtained according to the Animal Ethics Committee of the Federal University of Minas Gerais (protocol CEUA:31/2017). The teeth were stored in a $0.1 \%$ thymol solution for three months at most. Bovine incisors were selected over middle first teeth; those included showed external and 
internal anatomical similarity (length and diameter), with a completely formed dental apex and without root curvature. Bovine teeth with significant root curvature and/or with root fracture, and/or teeth with an open apex were excluded from the study. The crowns were removed using a diamond saw in a precision cutting machine (Isomet 1000, Buehler, Lake Bluff, USA), under constant water irrigation. The roots obtained were $15 \mathrm{~mm}$.

The sectioned teeth were radiographed to standardize the canal spaces, and the roots with similar canal diameters were selected. The canal aperture was tested with a Largo \#3 burr (Dentsply Sirona, Petrópolis, Brazil) to ensure selection of similar canal diameters. Only roots that showed some resistance to Largo \#3 drill penetration were used. One trained operator prepared the root canals. The canals were endodontically prepared using rotary instruments (S1,S2,F1,F2,F3,F4,F5; ProTaper Universal, Dentsply Sirona, Petrópolis, Brazil), under irrigation with $2.5 \%$ sodium hypochlorite (Asfer Indústria Química, São Caetano do Sul, Brazil). Final irrigation was performed with 17\% trisodium ethylenediamine tetra-acetic acid (EDTA; Biodinâmica, Ibiporã, Brazil) for 3 minutes, followed by rinsing with water and drying with absorbent paper points (\#40; Dentsply Sirona, Petrópolis, Brazil). The root canals were obturated using a gutta-percha thermoplastic technique with lateral and vertical condensation by hydraulic compression, and AH Plus sealer (Dentsply Sirona, Petrópolis, Brazil). They were then stored in $100 \%$ humidity at $37^{\circ} \mathrm{C}$ for 7 days..$^{12}$

\section{Post space preparation and post cementation}

The root canals were prepared using Largo burrs \#3 and \#4 (Dentsply Sirona, Petrópolis, Brazil) at a slow rotary speed, yielding a 10-mm-long apical canal, 4-mm of which was filled. The glass-fiber post used was Reforpost \#2 (Ângelus, Londrina, PR, Brazil). The teeth were randomly divided into four groups ( $n=3$ ) according to the protocol system used: water-wet bonding with dual cure resin cement (WBD); ethanol-wet bonding with dual cure resin cement (EBD); water-wet bonding with self-adhesive resin cement (WBS); and ethanol-wet bonding with self-adhesive resin cement (EBS). Randomization was achieved using random number sequences generated by the Microsoft Excel RAND function (Office Excel software; Microsoft Corp).

The fiber posts were immersed in 35\% hydrogen peroxide (FGM Produtos Odontológicos, Joinville, Brazil) for one minute, rinsed with water for one minute and dried with compressed air. ${ }^{13}$ Afterwards, a silane coupling agent was applied for one minute (Silano, Angelus, Londrina, Brazil). ${ }^{5}$ The roots were covered with black tape at the time of cementation, to prevent the influence of ambient light, and to create a condition similar to that of the oral environment.

In regard to the WBD groups, the root canals were rinsed with distilled water after etching with $37 \%$ phosphoric acid (FGM Produtos Odontológicos, Joinville, Brazil) for 15 seconds, and, in regard to the WBS groups, the root canals were rinsed with $2.5 \%$ sodium hypochlorite for 60 seconds, and the excess was removed from the post space using absorbent paper points. Regarding the EBD group, the root canals were rinsed with distilled water after etching, dried with sterile paper points, and completely filled with $100 \%$ ethanol for 60 seconds. Regarding the EBS group, the root canals were also rinsed with distilled water after etching, and dried with sterile points, but were rinsed with water after application of $2.5 \%$ sodium hypochlorite and dried with absorbent paper, after which they were completely filled with $100 \%$ ethanol for 60 seconds, and the excess ethanol was gently removed. ${ }^{2}$

After treating the root dentin, two cementation systems were evaluated (Table 1): an etch-and-rise 3-step adhesive system (Scotchbond Multipurpose Plus; 3M ESPE, St Paul, USA) with a dual cure resin cement clicker (RelyX ARC ${ }^{\mathrm{TM}}$, 3M ESPE, St Paul, USA), and a self-adhesive resin cement clicker (RelyXTMU200, 3M ESPE, St Paul, USA). The bonding agents and the resin cements were used according to the manufacturer's instructions (Table 1). The two types of resin cement were applied to the root canal with a syringe and a needle tip (Centrix; DFL).

All of the cement materials were light-activated for 40 seconds at each angulation (buccal, lingual and incisal), using a quartz-tungsten halogen light curer $\left(600 \mathrm{~mW} / \mathrm{cm}^{2}\right.$, Demetron 501; Kerr Corporation, Orange, USA) (12). After post cementation, the roots 
Table 1. Dual cure resin cements used.

\begin{tabular}{|c|c|c|c|}
\hline Resin cement & Composition & Manufacturer & Application instructions \\
\hline \multirow{8}{*}{$\begin{array}{l}\text { RelyX ARC/ Adper } \\
\text { Scotchbond } \\
\text { Multipurpose Plus }\end{array}$} & $\begin{array}{l}\text { Paste A: Silicon-treated ceramic, triethylene } \\
\text { glycol dimethacrylate (TEGDMA), bisphenol } \\
\text { A diglycidyl methacrylate (Bis-GMA); silane- } \\
\text { treated silica, functionalized dimethacrylate } \\
\text { polymer, 2-benzotriazolyl-4- methylphenol, } \\
\text { 4-(dimethylamino)-benzene ethanol }\end{array}$ & \multirow{8}{*}{ 3M ESPE } & \multirow[t]{8}{*}{$\begin{array}{l}\text { Mix the two pastes in a ratio of } 1: 1 \text { for } 10 \\
\text { seconds; apply mixture to root canal, and, after } \\
\text { slowly seating the post, light-activate for } 40 \\
\text { seconds in three different inclinations. Remove } \\
\text { excess cement before light-activating. }\end{array}$} \\
\hline & $\begin{array}{c}\text { Paste B: Silane-treated ceramic, TEGDMA, } \\
\text { Bis-GMA, silane-treated silica, functionalized } \\
\text { dimethacrylate polymer, 2-benzotriazolyl-4- } \\
\text { methylphenol, benzoyl peroxide }\end{array}$ & & \\
\hline & Primer: HEMA, polyalkenoic acid copolymer, water & & \\
\hline & \multirow[t]{5}{*}{$\begin{array}{c}\text { Adhesive: Bis-GMA, HEMA, tertiary amines, } \\
\text { photo-initiator }\end{array}$} & & \\
\hline & & & \\
\hline & & & \\
\hline & & & \\
\hline & & & \\
\hline \multirow[t]{2}{*}{ RelyX U200 } & $\begin{array}{c}\text { Base paste: Methacrylate monomers containing } \\
\text { phosphoric acid groups, methacrylate monomers, } \\
\text { silanated fillers, initiator components, stabilizers, } \\
\text { rheological additives }\end{array}$ & \multirow[t]{2}{*}{ 3M ESPE } & \multirow{2}{*}{$\begin{array}{l}\text { Mix the two pastes in a ratio of } 1: 1 \text { for } 10 \\
\text { seconds; apply mixture to root canal, and, after } \\
\text { slowly seating the post, light-activate for } 40 \\
\text { seconds in three different inclinations. Remove } \\
\text { excess cement before light-activating. }\end{array}$} \\
\hline & $\begin{array}{l}\text { Catalyst paste: Methacrylate monomers, alkaline } \\
\text { fillers, silanated fillers, initiator components, } \\
\text { stabilizers, pigments, rheological additives }\end{array}$ & & \\
\hline
\end{tabular}

were stored in water at room temperature for 7 days. During this period, the roots were submitted to micro-CT scanning and push-out testing.

\section{Micro-ct analysis}

After post cementation, the roots were mounted on a custom attachment and scanned using a micro-CT scanner (SkyScan 1272; Bruker-micro-CT, Kontich, Belgium). The scanner was operated at $100 \mathrm{kV}$ and $100 \mathrm{~mA}$ using a $0.11-\mathrm{mm} \mathrm{Cu}$ filter. The resolution used was $1224 \times 820$ pixels - $10 \mu \mathrm{m}$. The scanning was performed by $180^{\circ}$ rotation around the vertical axis with a rotation step of 0.6. The images of each root were reconstructed using NRecon software, version 1.6.3 (Bruker, micro-CT), to provide axial cross sections of the inner root structures, and the images were reconstructed with standardized digital lengths. CTAn software, version 1.12.0 (Brukermicro-CT), was used for the 3-dimensional analyses. The software analyzed the region of interest (area prepared for post cementation and cementation interface) to obtain the results, and then analyzed the 3-dimensional morphometry to provide the percentage of total porosity of the interfaces (\%) in the resin cement layer, as well as the interface with the root canal. ${ }^{5}$

\section{Push-out tests}

Bovine roots ( $n=3)$ were fixed in $20 \mathrm{~mm} \times 20 \mathrm{~mm}$ acrylic plates with sticky wax, and were sectioned perpendicular to the long fiber axis of the post into six slices for each root (approximate thickness of $1.0 \mathrm{~mm}$ ) using a precision cutting machine under water cooling (Isomet 1.000 Precision Saw, Buehler), resulting in two slices for each third. Each slice was marked on its apical side with an indelible marker, and the thickness was measured using digital calipers (Mitutoyo Digimatic Caliper Serie 500, Mitutoyo Sul Americana, Suzano, Brazil). The specimens were subjected to the push-out bond test with a $5000 \mathrm{~N}$ load cell, using a 1-mm-diameter plunger, and the load was applied at a crosshead speed of 
$0.5 \mathrm{~mm} / \mathrm{min}$ in the apical-coronal direction, using a universal testing machine (EZ-LX Long-Stroke Model, Kyoto, Japan). The plunger was centralized to avoid contact with the dentin. The bond strength was calculated in megapascals (MPa) by dividing the load recorded in newtons $(\mathrm{N})$ by the area $\left(\mathrm{mm}^{2}\right)$ of the post-dentin interface, which was calculated by using the following equation:

$$
\text { A- 2prh, }
$$

where $\mathrm{p}$ is $3.14, \mathrm{r}$ is the radius of the post (measured with a stereomicroscope), and $\mathrm{h}$ is the slice thickness in millimeters. ${ }^{2,12}$

The failure mode was observed using a stereomicroscope (Carl Zeiss AG) at 40X magnification. It was assessed by two calibrated operators independently, and calibration was performed to obtain optimum interexaminer agreement $($ Kappa $=0.85)$. Each sample was categorized as follows: adhesive failure (at the cement-dentin or cement-post interface), mixed, and cohesive failure (within the cement). ${ }^{2,12}$

\section{Post-gel shrinkage measurement}

The post-gel linear shrinkage was determined using the strain gauge method $(n=10)$ for each resin cement. ${ }^{14}$ Both resin cements were manipulated and placed on top of a biaxial strain gauge (CEA-06032WT-120, Measurements Group, Raleigh, USA), which measured the strains during polymerization in two perpendicular directions. The perpendicular strains were averaged, because the material properties were homogeneous and isotropic on the macro scale. Each resin cement was light-activated using the same light-curing unit used for sample preparation, with the light tip placed $1 \mathrm{~mm}$ from the surface of the cement. The radiant exposure was $24 \mathrm{~J} / \mathrm{cm}^{2}\left(600 \mathrm{~mW} / \mathrm{cm}^{2}\right)$ and was applied for 40 seconds. A strain conditioner (2101A Series, Micro Measurements Group) converted electrical resistance changes in the strain gauge into voltage changes, through a quarter-bridge circuit with an internal reference resistance. The post-gel strains that resulted from polymerization shrinkage were monitored for 10 minutes starting at the beginning of photoactivation..$^{14}$ The shrinkage strain, which is the linear post-gel shrinkage of each sample $(n=10)$, was converted into a percentage and multiplied by three to obtain the volumetric shrinkage.

\section{Statistical analysis}

The interface data and the values for push-out test and post-gel shrinkage were tested for normal distribution (Shapiro-Wilk, $\mathrm{p}>0.05$ ) and equality of variances (Levene test, $p>0.05$ ). The total porosity percentage and push-out test values were analyzed using two-way ANOVA and Tukey's post hoc test, according to the factors of pretreatment type (water-wet bonding and ethanol-wet bonding) and root third (coronal, middle, apical) under study. The post-gel shrinkage was evaluated using Student's $t$ test, and the data regarding interface integrity were analyzed using two-way ANOVA. Multiple comparisons were performed using Tukey's test. Pearson's correlation test was performed to analyze the relationship between the push-out bond strength and the void volume. All of the statistical analyses were performed using SPSS software, version 21.0 (SPSS, Chicago, USA), with a significance level of $\mathrm{a}=0.05$ for all of the tests.

\section{Results}

\section{Percentage of total porosity at the interface (\%)}

The mean volumes and standard deviations of the total porosity percentages are presented in Table 2 . Results of two-way ANOVA showed a difference for the pretreatment factor $(p<0.001)$; however, no significant difference was observed for the post space region $(p=0.968)$. The interaction between the factors of pretreatment and post space region $(p=0.209)$ was not significant. Tukey's test revealed that the percentage of total porosity was significantly lower for EBS for the coronal and apical thirds. The WBD group exhibited a higher percentage of total porosity for the coronal, middle and apical thirds.

\section{Push-out bond strength}

The means and standard deviations of the push-out bond values for all of the groups are presented in Table 3. Results of two-way ANOVA showed that a difference was observed for the pretreatment factor $(p<0.001)$; however, a significant interaction effect was observed $(p=0.001)$. The root canals prepared with EBD presented the best 
Table 2. Means \pm SD of total porosity at interface measured in coronal, middle, and apical thirds for pretreatment and resin cements.

\begin{tabular}{lcccc}
\hline & \multicolumn{3}{c}{ Pretreatment } \\
\cline { 2 - 5 } Location & RelyX ARC & RelyX U200 & EBS \\
\cline { 2 - 5 } & WBD & EBD & $0.62(0.02)^{c}$ & $0.50(0.01)^{d}$ \\
Coronal & $0.71(0.01)^{a}$ & $0.54(0.02)^{b}$ & $0.63(0.03)^{c}$ & $0.50(0.02)^{b}$ \\
Middle & $0.70(0.02)^{a}$ & $0.54(0.03)^{b}$ & $0.59(0.04)^{b}$ & $0.50(0.03)^{c}$ \\
\hline
\end{tabular}

Means followed by same superscript lowercase letters did not differ in comparisons by row. WBD: water-wet bonding with dual cure resin cement; EBD: ethanol-wet bonding with dual cure resin cement; WBS: water-wet bonding with self-adhesive resin cement; EBS: ethanol-wet bonding with self-adhesive resin cement.

Table 3. Means \pm SD of push-out bond strength values (MPa) measured at coronal, middle, and apical thirds for pretreatment and resin cements.

\begin{tabular}{lcccc}
\hline & \multicolumn{4}{c}{ Pretreatment } \\
\cline { 2 - 5 } Location & \multicolumn{2}{c}{ RelyX ARC } & \multicolumn{2}{c}{ RelyX U200 } \\
\cline { 2 - 5 } & WBD & EBD & WBS & EBS \\
\hline Coronal & $11.5(5.3)^{a}$ & $20.1(2.4)^{b}$ & $5.1(3.4)^{c}$ & $4.3(1.6)^{c}$ \\
Middle & $7.3(4.8)^{a}$ & $10.5(4.8)^{a}$ & $6.2(3.4)^{a}$ & $8.2(2.3)^{a}$ \\
Apical & $7.8(5.5)^{a}$ & $12.6(3.8)^{a, b}$ & $4.1(2.0)^{a, c}$ & $8.0(2.5)^{a}$ \\
\hline
\end{tabular}

Means followed by same superscript lowercase letters did not differ in comparisons by row. WBD: water-wet bonding with dual cure resin cement; EBD: ethanol-wet bonding with dual cure resin cement; WBS: water-wet bonding with self-adhesive resin cement; EBS: ethanol-wet bonding with self-adhesive resin cement

bonding performance for the coronal and apical thirds. The failure mode distribution is shown in Figure. Adhesive dentin-resin cement interface failure was the most frequent type of failure, irrespective of root dentin third.

There were no significant correlations between the push-out bond strength and the void volumes $(\mathrm{p}>0.05)$.

\section{Post-gel shrinkage values of resin cements}

The post-gel shrinkage values of the resin cements are presented in Table 4 . Student's $t$-test revealed that the post-gel shrinkage of RelyX ${ }^{\mathrm{TM}} \mathrm{ARC}$ differed significantly from that of RelyX'TM U200 ( $<<0.001)$.

\section{Discussion}

A hypothetical model was used to investigate the effects of root canal pretreatment on total porosity percentages at the interfaces, on bond strength of posts along the root canal, and on post-gel shrinkage. The use of ethanol-wet bonding promoted a better result for bubble volume and bond strength. The null hypothesis was partially rejected because the pretreatment used in the fixation of fiber posts in the root canals influenced the total porosity and bond strength, and the resin cement type influenced the degree of post-gel shrinkage. However, the root canal factor was not influenced by the region.

Micro-CT is a high-resolution method able to measure the volumes of gaps and voids around the interface in three dimensions (3D). ${ }^{15}$ Micro-CT systems using microfocus spot $\mathrm{X}$-ray sources and high-resolution detectors allow projections to rotate through multiple viewing directions to produce 3D reconstructed images of samples. ${ }^{5}$ Furthermore, they offer the advantage of being a nondestructive method that can analyze all the specimen samples, ${ }^{16}$ and still be available for additional bond testing (reuse). ${ }^{5}$ It is important to evaluate the volume of voids in the cementation of glass-fiber posts for clinical situations, because the presence of voids can affect the longevity of adhesive bonding in three ways: voids located at the interface between the dentin and the cement can decrease the contact bonded area; the presence of voids can decrease the strength of the cement and create sites for crack initiation and propagation, ${ }^{16}$ and the presence of bubbles acts as a stress concentration factor that reduces the bond area, and reflects the highest stress peak on the interface. ${ }^{5}$ In this study, micro CT identified lower porosity at the interface of ethanol-wet bonding with RelyX ${ }^{\mathrm{TM}}$ U200. Water-wet bonding pretreatment obtained high values of porosity, independent of the type of 


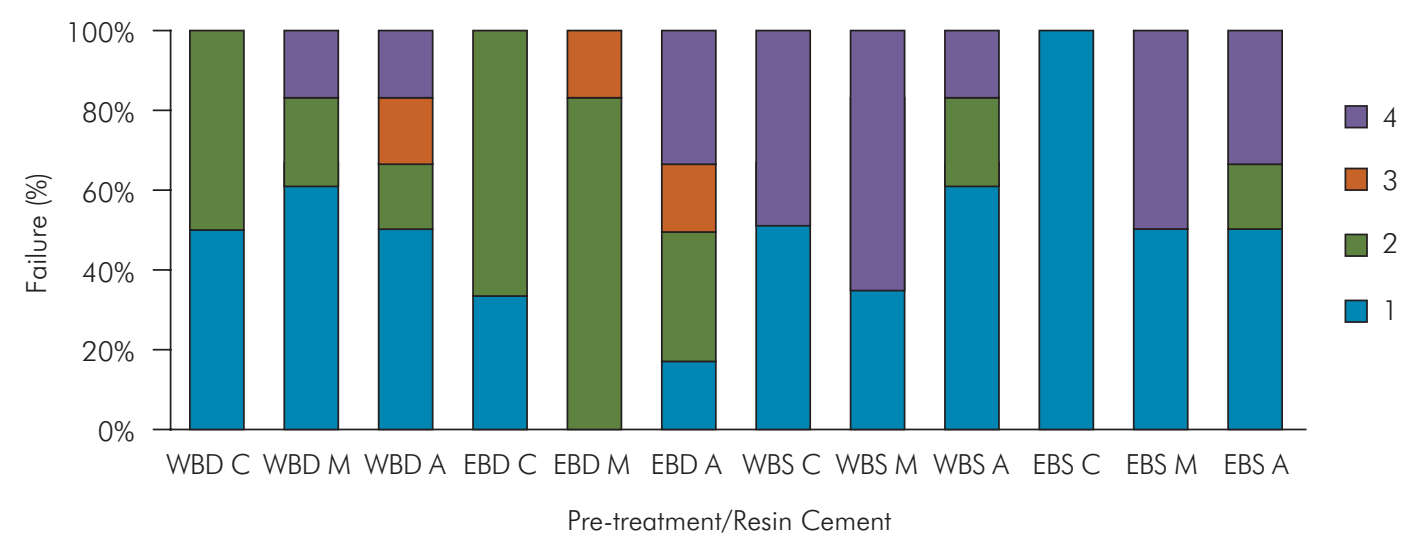

1: adhesive failure; 2: cohesive failure in cement; 3 : cohesive failure in dentin; 4: mixed failure. Bottom legends refer to pretreatment and resin cement for root levels. WBD C: Water-wet bonding with RelyX ${ }^{T M}$ ARC in the coronal third; WBD M: Water-wet bonding with RelyX $X^{T M}$ ARC in the middle third; WBD A: Water-wet bonding with RelyX $X^{T M}$ ARC in the apical third; EBD C: Ethanol-wet bonding with RelyX ${ }^{T M} A R C$ in the coronal third; EBD M: Ethanol-wet bonding with RelyX $X^{T M} A R C$ in the middle third; EBD A: Ethanol-wet bonding with RelyX $X^{\text {TM }}$ ARC in the apical third; WBS C, Water-wet bonding with RelyX ${ }^{\mathrm{TM}}$ U200 in the coronal third; WBS M: Water-wet bonding with RelyX $X^{\mathrm{TM}}$ U200 in the middle third; WBS A: Water-wet bonding with RelyX ${ }^{T M}$ U200 in the coronal and the apical thirds; EBS C: Ethanol-wet bonding with RelyX ${ }^{T M}$ U200 in the coronal third; EBS M: Ethanol-wet bonding with RelyX ${ }^{T M}$ U200 in the middle third; EBS A: Ethanol-wet bonding with RelyX ${ }^{T M}$ U200 in the apical third.

Figure. Failure modes found in different pretreatment and resin cement systems for different root levels.

Table 4. Means \pm SD for post-gel shrinkage of the two resin cements.

\begin{tabular}{lccc}
\hline Resin cements & RelyX $X^{\top M}$ ARC & RelyX $^{\top M} \cup 200$ & p-value* \\
\hline $\begin{array}{l}\text { Post-gel } \\
\text { shrinkage }\end{array}$ & $0.97 \pm 0.15 \%$ & $0.77 \pm 0.026 \%$ & 0.001 \\
\hline
\end{tabular}

resin cement. These results could be explained by the immiscibility of different solutions. Specifically, the incompatibility of hydrophobic resin and water causes the two liquids to become immiscible due to the high value of total cohesive forces, pointing out that this value was different when water was replaced with $100 \%$ ethanol. ${ }^{10}$ When comparing RelyX ${ }^{\mathrm{TM}} \mathrm{U} 200$ and RelyX ${ }^{\mathrm{TM}}$ ARC with ethanol-wet bonding, the lower porosity of RelyX ${ }^{\mathrm{TM}} \mathrm{U} 200$ might be explained by the lower degree of post-gel shrinkage. ${ }^{1}$ These findings suggest a possible relationship between polymerization shrinkage and porosity percentage.

Making use of tips to insert the resin cement into the root canal renders the technique more predictable, because fewer bubbles and voids are formed. ${ }^{17}$ However, void formation might also be the result of air entrapment in the material during the mixing process. ${ }^{15}$ Indeed, as regards fluid material, voids may be formed indirectly when small bubbles interconnect. Thus, the presence of bubbles does not depend on the type of resin cement used. Noteworthily, the types of pores identified in resin cements are classified as mesoporous, meaning that they have diameters smaller than 20 nanometers. ${ }^{18}$ The reason why the porosity was not significantly different among the intragroup root thirds may be attributed to the insertion of resin cement using a Centrix syringe in the root canal, a device that promotes a more homogeneous distribution of the material. A similar study found that the air bubbles do not derive from a fault in the luting procedure, but from the mixing phase of the substrate paste with the catalyst. Indeed, control of the variables, such as the diameter of the root canal and the root preparation, promoted greater proximity to the cementation line. ${ }^{17}$ This outcome can be explained by the difference in the degree of post-gel contraction, and by the thin and uniform cement layers at the cementing line, yielding lower

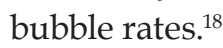

The push-out test used in the present investigation to measure bond strength is a more appropriate methodology to evaluate fiber post adhesion in root dentin. ${ }^{2,5}$ The main aspect of the push-out test that differentiates it from tensile and shear tests in flat bonded interfaces is the confinement of the composite within the walls of the prepared cavity, a condition more akin to that found in a clinical setting. ${ }^{19}$ 
Furthermore, the possibilities of premature failure are reduced, compared with tests like the trimming and non-trimming microtensile tests. In performing the push-out test, some parameters must be observed, such as the size and diameter of the orifice base, and the thickness of the specimens. ${ }^{19}$

The dentin matrix is composed mainly of type I collagen and non-collagenous proteins, and the fibrils are intrinsically wet, because of their high affinity with water. Adhesive components are more prone to degradation over time, because of water sorption and resin leaching. Initially, ethanol-wet bonding was performed with a progressive ethanol substitution technique used to chemically dehydrate the demineralized collagen matrix. ${ }^{8}$ Shrinkage of the collagen fibrils in their lateral dimension was likely the result of the development of intrafibrillar hydrogen bonding within individual collagen fibrils, since ethanol has a lower capacity to break these hydrogen bonds than water. ${ }^{8}$ Increases in interfibrillar volume are caused by the disruption of bridges that maintain the shape of the interfibrillar proteoglycan..$^{20}$ However, extensive clinical application has resulted in the development of a simplified technique. ${ }^{2}$ The principles of ethanol-wet bonding might be explained using Hoy's triple solubility parameter theory, which consists of dispersive forces ( $\delta \mathrm{d})$, polar forces $(\delta p)$, hydrogen bonding forces ( $\delta \mathrm{h})$, and total cohesive forces $(\delta \mathrm{t}) \cdot{ }^{10}$ Solvents require a higher hydrogen bonding force than $14.8\left(\mathrm{~J} / \mathrm{cm}^{3}\right)$ to re-expand matrices. Water-wet bonding is capable of re-expanding a matrix, since water has the very high $\delta$ h value of 40 $\left(\mathrm{J} / \mathrm{cm}^{3}\right)$. However, not all monomers are soluble in water. ${ }^{8}$ Therefore, ethanol could be an alternative to water, because the ethanol $\delta \mathrm{h}(\mathrm{EtOH})$ value is 20.0 $\mathrm{J} / \mathrm{cm}^{3}$, entailing limited collapse of matrices and high resin infiltration in the hybrid layer. ${ }^{10}$ Another issue concerns the presence of proteoglycan gel in interfibrillar spaces in the dentin matrix. EtOH can cause these hydrogels to collapse, whereas ethanol should remain in close contact with collagen, thus bringing the monomers into direct contact with the matrix. ${ }^{20}$ The results of this study identified a high value for bond strength to ethanol-wet bonding with RelyX ARC ${ }^{\mathrm{TM}}$ for the coronal third. These results can be explained by the ability of $\mathrm{EtOH}$ to produce interpeptide hydrogen bonding, and to maintain the interfibrillar spaces, which serve as diffusion channels for resin infiltration. ${ }^{8}$ The study by Carvalho et al. (2009) ${ }^{9}$ detected that collagen fibril diameters in ethanol-saturated dentin were smaller than those in water-saturated dentin matrices, thus leaving larger interfibrillar spaces available for resin impregnation. Because higher bond strength is correlated to wider interfibrillar spaces, the use of ethanol can increase bond strength.

Challenges are imposed when bonding fiber posts to the root canal, especially in the apical area, with an adhesive system and polymerization light access. ${ }^{16}$ Some of the difficulties include a smaller number of dentinal tubules and a greater likelihood of presenting endodontic treatment remnants. ${ }^{4}$ Another consideration is the configuration factor (C-factor), which is the ratio of the bonded to the unbonded surface areas of cavities. This factor is very high in root dentin due to the limited amount of unbonded surface area, compared with the area of the bonded surface; hence, the chances for debonding are very high. ${ }^{2}$ In addition, the deeper portions may be inaccessible to light, and a lower degree of conversion in the apical portion may affect the mechanical properties of resin cement. ${ }^{12}$ Despite the difficulties in post cementation to the cervical from the apical thirds, the study found no significant differences among the root thirds. The authors of the present study corroborate the results found by Ekabaram et al., ${ }^{2}$ whose analysis of the data found that the bond strength was not affected by the root third factor, unlike the results by Bitter et al., ${ }^{6}$ who observed a significant reduction in bond strength in the middle and apical portions of the root canal.

Voids in continuity from the coronal to the apical level of the interface can cause easier dissolution of the resin cement and dislodgement of post-core restorations under functional stresses. Another negative effect of the formation of voids is that they decrease the bonding strength by restricting the available area for cementation, resulting in shortened survival time of restorations. ${ }^{15}$ However, the present study found no correlation between void volumes and push-out bond strength data, in agreement with the findings of previous investigations..$^{15}$ The presence of a thicker smear layer in the apical third and more 
remnants of endodontic sealer and gutta-percha in the cervical and middle thirds may have resulted in more open dentinal tubules for micromechanical bonding. ${ }^{15}$ These factors can diminish the negative effects of void parameters on bond strength. However, Silva et al. ${ }^{25}$ found that bond strength was affected by void volumes, independent of the region of the root. Indeed, the authors identified that the presence of bubbles and voids promoted stress concentrations at the resin cement-dentin interface and reduced the fiber post bond strength.

Improvements in the adherence of adhesive systems to dental tissues has prompted a rise in the frequency of cohesive failures during bond strength testing. This failure precludes determining the real interfacial bond strength, ${ }^{21}$ thus highlighting the importance of assessing the failure modes of specimens. The most common failure modes observed in this study were adhesive failure between the dentin and the resin cement, and between the post and the resin cement, pointing out that the interfaces at these locations experience the greatest stress concentrations, and are the most suitable areas for glass-fiber post cementation. This result corroborates that of another report indicating that these interfaces were expected to have lower bond strength. ${ }^{18}$ However, it was demonstrated that etching the fiber post with hydrogen peroxide improved the bonding of the resin to the glass fiber. ${ }^{13}$ Nevertheless, ethanol-wet bonding with RelyX ARC ${ }^{\mathrm{TM}}$ showed the most cohesive failures. The high bond strength for this group might have been responsible for this type of failure. ${ }^{7}$

Composite restoratives have the inherent property of polymerization shrinkage, which produces a volumetric change and distributes contractile stresses. ${ }^{14}$ This shrinkage can be divided into two phases relative to the development of the elastic modulus. In the pre-gel phase, the composites are able to flow, relieving stress within the restoration. After gelation, the composite develops shrinkage stresses because of the advancement of the elastic modulus. Internal shrinkage stresses occur because flow is not able to compensate for the contraction. ${ }^{14}$ Therefore, possible occurrence of shrinkage stress and post-gel shrinkage was related, as confirmed by Soares et al., ${ }^{22}$ who found a strong correlation between the two factors. The present study found a significant difference between the two resin cements, corroborating Braga et al., ${ }^{23}$ who explained that these differences depended on the characteristics of the polymer network and the overall composition of the resin cement. Post-gel contraction is characterized by the development of internal forces in the material, resulting in molecular rearrangement (viscoelastic properties), and by the inability of the material to compensate for polymerization shrinkage by plastic flow and deformation. ${ }^{24}$ The magnitude of volumetric shrinkage experienced by a composite is determined by its filler volume fraction and by the composition and degree of conversion of the resin matrix. ${ }^{23}$ Higher postgel shrinkage was obtained with RelyX ${ }^{\mathrm{TM}}$ ARC resin cement $(0.97 \%)$ than with RelyX ${ }^{\mathrm{TM}} \mathrm{U} 200$ cement $(0.77 \%)$. This result might be explained by the difference in the composition of the materials, resulting in variations in the volumetric shrinkage. According to the manufacturer's information, Rely $\mathrm{X}^{\mathrm{TM}} \mathrm{ARC}$ resin cement has bisphenol A diglycidyl methacrylate (Bis-GMA) and triethylene glycol dimethacrylate (TEGDMA) as monomers, and RelyX ${ }^{\mathrm{TM}} \mathrm{U} 200$ has more TEGDMA. Bis-GMA has a greater number of covalent bonds, and thus better monomer conversion and greater post-gel polymerization..$^{25}$ According to de Souza et al., ${ }^{25}$ acidic monomers have been found to negatively affect the cement degree of conversion. For this reason, differences in volume fraction, ty pe of resin cement, molecular weight and functionality might explain the results regarding their study versus ours. However, a lower degree of conversion influences certain mechanical properties, such as bond strength. The degree of conversion was inversely proportional to the concentration of base monomers. The exception was pure TEGDMA, which exhibited a low conversion of $42 \%$, and Bis-GMA-containing polymers, which had a conversion rate ranging from $54 \%$ to $85 \%{ }^{26}$ However, their study indicated that increasing the diluent monomer concentration decreases the leachable fraction. Other factors that could be influenced by bond strength are the elastic modulus and the Vickers hardness. The Vickers hardness and the degree of conversion of resin cements are directly related, whereas the elastic modulus is directly related to Vickers hardness and polymerization stress. ${ }^{1}$ These 
factors might explain why RelyX ${ }^{\mathrm{TM}} \mathrm{U} 200$ had the lowest bond strength values.

The present study was devised to evaluate simplified ethanol-wet bonding in adhesive cementation for fiber posts, but the results of in vitro studies should be carefully interpreted before extrapolating them to the clinical context. Further research should be performed with a representative sample to improve the results for the mechanical proprieties.

\section{Conclusion}

According to the methodology employed, and within the limitations of this study, it can be concluded that pretreatment with ethanol-wet bonding performed better in adapting glass-fiber posts to the root canal, and self-adhesive resin cement showed the best values for porosity and post-gel shrinkage.

\section{Acknowledgements}

This project was funded by CNPq (National Council for Scientific and Technological Development), and FAPEMIG (Fundação de Amparo à Pesquisa do Estado de Minas Gerais) (APQ01837-16). The authors would like to thank CPBioUFU (Uberlândia/MG/Brasil) for its availability and partnership.

\section{References}

1. Pereira RD, Valdívia AD, Bicalho AA, Franco SD, Tantbirojn D, Versluis A, et al. Effect of Photoactivation timing on the mechanical properties of resin cements and bond strength of fiberglass post to root dentin. Oper Dent. 2015 Sep-Oct;40(5):E206-21. https://doi.org/10.2341/14-115-L

2. Ekambaram M, Yiu CK, Matinlinna JP, Chang JW, Tay FR, King NM. Effect of chlorhexidine and ethanol-wet bonding with a hydrophobic adhesive to intraradicular dentine. J Dent. 2014 Jul;42(7):872-82. https://doi.org/10.1016/j.jdent.2014.02.006

3. Sarkis-Onofre R, Skupien JA, Cenci MS, Moraes RR, Pereira-Cenci T. The role of resin cement on bond strength of glass-fiber posts luted into root canals: a systematic review and meta-analysis of in vitro studies. Oper Dent. 2014 Jan-Feb;39(1):E31-44. https://doi.org/10.2341/13-070-LIT

4. Daleprane B, Pereira CN, Bueno AC, Ferreira RC, Moreira AN, Magalhães CS. Bond strength of fiber posts to the root canal: effects of anatomic root levels and resin cements. J Prosthet Dent. 2016 Sep;116(3):416-24. https://doi.org/10.1016/i.prosdent.2016.01.030

5. Silva NR, Aguiar GC, Rodrigues MP, Bicalho AA, Soares PB, Veríssimo C, et al. Effect of resin cement porosity on retention of glass-fiber posts to root dentin: an experimental and finite element analysis. Braz Dent J. 2015 Nov-Dec;26(6):630-6. https://doi.org/10.1590/0103-6440201300589

6. Bitter K, Aschendorff L, Neumann K, Blunck U, Sterzenbach G. Do chlorhexidine and ethanol improve bond strength and durability of adhesion of fiber posts inside the root canal? Clin Oral Investig. 2014 Apr;18(3):927-34. https://doi.org/10.1007/s00784-013-1040-1

7. Bitter K, Polster L, Askar H, Stein-Lausnitz M, Sterzenbach G. Effect of final irrigation protocol and etching mode on bond strength of a multimode adhesive in the root canal. J Adhes Dent. 2017 Jun;245-52. https://doi.org/10.3290/i.jad.a3841

8. Pashley DH, Tay FR, Carvalho RM, Rueggeberg FA, Agee KA, Carrilho M, et al. From dry bonding to water-wet bonding to ethanol-wet bonding.: a review of the interactions between dentin matrix and solvated resins using a macromodel of the hybrid layer. Am J Dent. 2007 Feb;20(1):7-20.

9. Carvalho CA, Cantoro A, Mazzoni A, Goracci C, Breschi L, Ferrari M. Effect of ethanol application on post-luting to intraradicular dentine. Int Endod J. 2009 Feb;42(2):129-35. https://doi.org/10.1111/i.1365-2591.2008.01491.x

10. Sadek FT, Pashley DH, Nishitani Y, Carrilho MR, Donnelly A, Ferrari M, et al. Application of hydrophobic resin adhesives to acid-etched dentin with an alternative wet bonding technique. J Biomed Mater Res A. 2008 Jan;84(1):19-29. https://doi.org/10.1002/jbm.a.31290

11. Aguiar TR, Oliveira M, Arrais CA, Ambrosano GM, Rueggeberg F, Giannini M. The effect of photopolymerization on the degree of conversion, polymerization kinetic, biaxial flexure strength, and modulus of self-adhesive resin cements. J Prosthet Dent. 2015 Feb;113(2):128-34. https://doi.org/10.1016/i.prosdent.2014.09.011

12. Daleprane B, Pereira CNB, Oréfice RL, Bueno AC, Vaz RR, Moreira AN, et al. The effect of light-curing access and different resin cements on apical bond strength of fiber posts. Oper Dent. 2014 Mar-Apr;39(2):E93-100. https://doi.org/10.2341/12-477-L

13. Menezes MS, Queiroz EC, Soares PV, Faria-e-Silva AL, Soares CJ, Martins LR. Fiber post etching with hydrogen peroxide: effect of concentration and application time. J Endod. 2011 Mar;37(3):398-402. https://doi.org/10.1016/i.joen.2010.11.037 
14. Sakaguchi RL, Sasik CT, Bunczak MA, Douglas WH. Strain gauge method for measuring polymerization contraction of composite restoratives. J Dent. 1991 Oct;19(5):312-6. https://doi.org/10.1016/0300-5712(91)90081-9

15. Uzun I, Keleş A, Arslan H, Güler B, Keskin C, Gündüz K. Influence of oval and circular posł placement using different resin cements on push-out bond strength and void volume analysed by micro-CT. Int Endod J. 2016 Dec;49(12):1175-82. https://doi.org/10.1111/iej.12568

16. Lorenzoni FC, Bonfante EA, Bonfante G, Martins LM, Witek L, Silva NR. MicroCT analysis of a retrieved root restored with a bonded fiber-reinforced composite dowel: a pilot study. J Prosthodont. 2013 Aug;22(6):478-83. https://doi.org/10.1111/jopr.12045

17. Boschian Pest L, Cavalli G, Bertani P, Gagliani M. Adhesive post-endodontic restorations with fiber posts: push-out tests and SEM observations. Dent Mater. 2002 Dec;18(8):596-602. https://doi.org/10.1016/S0109-5641(02)00003-9

18. D'Arcangelo C, Cinelli M, De Angelis F, D'Amario M. The effect of resin cement film thickness on the pullout strength of a fiber-reinforced post system. J Prosthet Dent. 2007 Sep;98(3):193-8. https://doi.org/10.1016/S0022-3913(07)60055-9

19. Boaro LC, Fróes-Salgado NR, Gajewski VE, Bicalho AA, Valdivia AD, Soares CJ, et al. Correlation between polymerization stress and interfacial integrity of composites restorations assessed by different in vitro tests. Dent Mater. 2014 Sep;30(9):984-92. https://doi.org/10.1016/j.dental.2014.05.011

20. Tay FR, Pashley DH, Kapur RR, Carrilho MR, Hur YB, Garrett LV, et al. Bonding BisGMA to dentin —a proof of concept for hydrophobic dentin bonding. J Dent Res. 2007 Nov;86(11):1034-9. https://doi.org/10.1177/154405910708601103

21. Giannini M, Soares CJ, Carvalho RM. Ultimate tensile strength of tooth structures. Dent Mater. 2004 May;20(4):322-9. https://doi.org/10.1016/S0109-5641(03)00110-6

22. Soares CJ, Bicalho AA, Tantbirojn D, Versluis A. Polymerization shrinkage stresses in a premolar restored with different composite resins and different incremental techniques. J Adhes Dent. 2013 Aug;15(4):341-50.

23. Braga RR, Ferracane JL, Condon JR. Polymerization contraction stress in dual-cure cements and its effect on interfacial integrity of bonded inlays. J Dent. 2002 Sep-Nov;30(7-8):333-40. https://doi.org/10.1016/S0300-5712(02)00047-7

24. Pereira R, Bicalho AA, Franco SD, Tantbirojn D, Versluis A, Soares CJ. Effect of restorative protocol on cuspal strain and residual stress in endodontically treated molars. Oper Dent. 2016 Jan-Feb;41(1):23-33. https://doi.org/10.2341/14-178-L

25. Silva NR, Aguiar GC, Rodrigues MP, Bicalho AA, Soares PB, Veríssimo C, et al. Effect of resin cement porosity on retention of glass-fiber posts to root dentin: an experimental and finite element analysis. Braz Dent J. 2015 Nov-Dec;26(6):630-6. https://doi.org/10.1590/0103-6440201300589

26. Floyd CJ, Dickens SH. Network structure of Bis-GMA- and UDMA-based resin systems. Dent Mater. 2006 Dec;22(12):1143-9. https://doi.org/10.1016/i.dental.2005.10.009 